\title{
SUPLEMENTAÇÃO DE LECITINA DE SOJA NA RAÇÃO DE CÃES: estudo sobre o perfil hematológico
}

\author{
Dog food supplementation with soy lecithin: study on the bematologic profile
}

\author{
Solange Aparecida Marconcin ${ }^{[a]}$, Palloma Rose ${ }^{[b]}$, Lia Lenati ${ }^{[c]}$, \\ Ana Vitória Fischer da Silva ${ }^{[\mathrm{d}]}$
}

\footnotetext{
[a] Médica Veterinária, Mestre em Ciências Veterinárias pela Universidade Federal do Paraná (UFPR), Curitiba, PR - Brasil, e-mail: solmarconcin@yahoo.com

${ }^{[b]}$ Mestranda do Curso de Pós-Graduação em Ciências Veterinárias, Departamento de Medicina Veterinária da Universidade Federal do Paraná (UFPR), Curitiba, PR - Brasil, e-mail: dove_vet@hotmail.com

${ }^{[c]}$ Mestranda do Curso Pós-Graduação em Ciências Veterinárias, Departamento de Medicina Veterinária da Universidade Federal do Paraná (UFPR), Curitiba, PR - Brasil, e-mail: lialenati@gmail.com

[d] Professora titular do Departamento de Fisiologia, Setor de Ciências Biológicas da Universidade Federal do Paraná (UFPR), Curitiba, PR - Brasil, e-mail: avitoria@ufpr.br
}

\begin{abstract}
Resumo
Com o objetivo de avaliar os efeitos da suplementação de lecitina de soja sobre o perfil hematológico, 8 cães da raça Beagle foram divididos de forma aleatória em grupo controle (C) e grupo suplementado com $2 \mathrm{~g} / \mathrm{kg}$ de lecitina de soja (LS) na ração e avaliados por um período de 60 dias. As coletas de sangue para os ensaios hematológicos foram feitas nos dias 1, 30 e 60, com os animais em jejum de 14 horas. Os dados foram analisados utilizando o programa estatístico Estat ${ }^{\circledR}$ 2.0, com o delineamento inteiramente casualizado, adotando o esquema fatorial $2 \times 2 \times 3$. Os resultados não mostraram diferenças da ação do aditivo sobre o número de eritrócitos, leucócitos totais, neutrófilos segmentados e bastonetes, linfócitos e monócitos. Os resultados obtidos neste estudo constituem o primeiro relato da ação da lecitina de soja em cães, portanto, mais estudos são necessários para avaliar sua real efetividade.
\end{abstract}

Palavras-chave: Cães. Hematologia. Lecitina de soja.

\section{Abstract}

The aim of this work was to evaluate the effects of dietary soybean lecithin on the hematologic profile of 8 adult healthy beagles, divided randomly into 2 groups: $C$ - control group and LS - group supplemented with $2 \mathrm{~g} / \mathrm{kg}$ of soybean lecithin, evaluated for a 60 days period. Blood samples were collected at day 1, 30 and 60, with the animals fasted for 14 hours. Statistical analysis was performed using Estat ${ }^{\circledR} 2.0$ and a $2 \times 2 \times 3$ factorial analysis. The 
hematological profile of the supplemented group showed no alterations in total erytrocytes count, total leucocytes count, neutrophil, lymphocytes and monocytes counts considering that the results found in this study represent the first report about the effects of soybean lecithin in dogs, further studies in these field are required to evaluate its real effectivity.

\section{Keywords: Dogs. Hematology. Soyabean lecithin.}

\section{INTRODUÇÃO}

A lecitina é uma mistura de fosfolipídios (fosfatidilcolina, fosfatidilinositol e fosfatidiletanol), triacilgliceróis e glicolipídios presente em diversas fontes vegetais e animais, como soja e outros grãos, gérmen de trigo, peixe e fígado (GORMLEY, 1997).

De todos os fosfolipídios presentes na lecitina, a fosfatidilcolina é singularmente o mais importante. Sua molécula é composta por ácidos graxos esterificados a uma molécula de glicerol-3fosfato e colina, servindo como fonte principal deste nutriente, importante para a função hepática, usado na síntese dos componentes estruturais de todas as membranas celulares, na sinalização molecular e como fator de ativação plaquetária. A colina também é precursora de um importante neurotransmissor, a acetilcolina, envolvida principalmente no controle muscular (MILLER, 2002).

Alguns autores afirmaram que o alto consumo de soja e de seus subprodutos pode reduzir o risco de câncer (NAGATA, 2000; NAGATA et al., 2002) e a incidência de doenças cardiovasculares (ITO et al., 2003; ZHANG et al., 2003). Dietas contendo fosfatidilcolina reduziram o número de nódulos tumorais no fígado de ratos, conservando a função hepática desses animais (SAKAKIMA et al., 2007).

A fosfatidilcolina também pode interferir no metabolismo do colesterol e outros lipídios, pois exerce um importante papel na absorção intestinal de lipídios por elevar a solubilidade das micelas lipídicas e fornecer revestimento da superfície para a formação de quilomicrons, além de alterar a composição dos depósitos de gordura, removendo colesterol dos tecidos (STRAYER, 1996; YONGZHI et al., 2001). Lechowski et al. (1999) verificaram que roedores suplementados com lecitina durante 36 dias tiveram redução significativa das concentrações de colesterol total, triacilgliceróis e de HDL. Já em macacos, Wilson, Meservey e Nicolos (1998) notaram que após 8 semanas de suplementação com lecitina, houve diminuição das concentrações de colesterol e de LDL, mas nenhum efeito sobre a de HDL.

Leblanc et al. (2003) observaram que a adição de lecitina a roedores durante 14 dias estimulou a secreção de ácido biliar, fosfolipídios e colesterol na bile, e inibiu a enzima ACAT (colesterol - aciltransferase), responsável por distribuir o colesterol nos hepatócitos. Quando esta enzima é inibida, o colesterol é direcionado à bile para sua eliminação. Mastellone et al. (2000) encontraram aumento das concentrações de sais biliares, fosfolipídios e colesterol na bile de coelhos suplementados com lecitina de soja.

A lecitina de soja também apresenta propriedades antioxidantes. Foi relatado por Aabdallah e Eid (2004) um efeito neuroprotetor da lecitina, devido em parte a esta ação, que protege tanto os ácidos graxos poliinsaturados, evitando sua oxidação e produção de radicais livres, como as células de todo o organismo.

Atribui-se aos ácidos graxos poliinsaturados da lecitina de soja, especialmente ao ácido linoleico, a renovação dos tecidos e a regeneração das células por estimular a divisão celular, assim seu uso tópico tem indicação para proteção, hidratação e cicatrização da pele (DECLAIR, 1994). Os fosfolipídios são componentes importantes das membranas celulares do organismo, incluindo as células sanguíneas, contribuindo na sua integridade estrutural, assim como na reparação dos tecidos (STRAYER, 1996).

O sangue é composto por uma parte líquida - o plasma, formado por água, sais minerais, moléculas orgânico-proteicas e por elementos celulares: hemácias ou eritrócitos, leucócitos e plaquetas (BEVILACQUA et al., 1992). A análise da série vermelha é constituída pela contagem de eritrócitos, dosagem da hemoglobina ( $\mathrm{Hb})$, hematócrito $(\mathrm{Ht})$, volume corpuscular médio (VCM), hemoglobina corpuscular média (HCM) e concentração da hemoglobina corpuscular média (CHCM). A série branca,

Rev. Acad., Ciênc. Agrár. Ambient., Curitiba, v. 7, n. 3, p. 289-297, jul./set. 2009 
por sua vez, é analisada por meio da contagem total de leucócitos e contagem diferencial de leucócitos: neutrófilos (bastonetes e segmentados), eosinófilos, basófilos, linfócitos e monócitos (GARCIANAVARRO, 2005). A contagem de plaquetas, células responsáveis pelo processo de hemostasia, e a dosagem de proteínas plasmáticas, em especial do fibrinogênio, proteína plasmática importante na coagulação sanguínea e na inflamação, também fazem parte da avaliação hematológica de rotina na medicina veterinária (THRALL; WEISER, 2006).

Os eritrócitos são produzidos na medula óssea e respondem por $40 \%$ de todo o volume sanguíneo. Por meio da hemoglobina se faz o transporte do $\mathrm{O}_{2}$ dos pulmões para os tecidos e de $\mathrm{CO}_{2}$ no sentido inverso (GARCIA-NAVARRO, 2005). O eritrócito do cão é um disco bicôncavo anucleado, com área central notadamente pálida, quando visualizada em esfregaços sanguíneos corados. A duração média da vida dos eritrócitos caninos é de 120 dias (DUNCAN; PRASSE, 1982).

Os leucócitos são as células brancas do sangue, formadas na medula óssea e compreendem os granulócitos (neutrófilos, eosinófilos e basófilos), monócitos e linfócitos (SODIKOFF, 2001); desempenham atividade nos processos inflamatório e imunológico dos tecidos por meio da quimiotaxia, fagocitose, amebismo e diapedese (BEVILACQUA et al., 1992).

Os neutrófilos são os leucócitos que aparecem em maior número no esfregaço sanguíneo. Possuem núcleo segmentado, unidos por filamentos de cromatina e grânulos citoplasmáticos (CARR; RODAK, 2000). A segmentação do núcleo acompanha o envelhecimento da célula, assim os neutrófilos jovens têm o núcleo em forma de bastão não segmentado e são chamados de bastonetes (GARCIANAVARRO, 2005). Suas principais funções incluem a fagocitose e a atividade bactericida, desempenhadas por meio da quimiotaxia e migração pela parede vascular (DUNCAN; PRASSE, 1982). Também elaboram poderosas enzimas proteolíticas que podem agir dentro da célula para a destruição de partículas fagocitadas, ou serem liberadas e atuar fora do corpo celular (THRALL; WEISER, 2007). Por serem a primeira linha de defesa do sistema imunológico (SODIKOFF, 2001), é de fundamental importância sua constante síntese pela medula óssea, o que demanda grandes quantidades de substrato. A lecitina, por estimular a divisão celular, fornecendo fosfolipídios, tem papel relevante na manutenção da integridade das membranas (STRAYER, 1996), ajudando as células a executarem com eficiência suas funções.

Os monócitos são os maiores leucócitos circulantes, apresentam intensa capacidade fagocítica e representam a segunda linha de defesa do sistema fagocítico circulante (SINK; FELDMAN, 2006). Transmigram entre células endoteliais por diapedese e alojam-se em outros tecidos, onde recebem diferentes nomes de acordo com a localização: histiócitos (tecido conjuntivo e músculos), células de Kupffer (fígado), microglia (cérebro), macrófagos alveolares (pulmão), células de Langherans (pele), osteoclastos (tecido ósseo). Os macrófagos, dentro das lesões inflamatórias crônicas, se diferenciam em células epiteliloides ou podem unir-se para formar células gigantes de corpo estranho ou células gigantes de Langhans (JAIN, 1993; THRALL; WEISER, 2007).

Basófilos originam-se da célula precursora mieloide, a mesma célula progenitora dos mastócitos (CARR; RODAK, 2000). São células grandes que apresentam o núcleo redondo com muitos grânulos metacromáticos, são raras no sangue e na medula óssea de cães (JAIN, 1993). A função precisa dos basófilos ainda não foi delineada, mas sabe-se que seus grânulos contêm heparina e histamina, importantes mediadores na modulação da resposta inflamatória, nas reações de hipersensibilidade imediata e na inibição do mecanismo de coagulação (SINK; FELDMAN, 2006). Alterações na membrana plasmática destas células podem comprometer a resposta ante os antígenos (CALDER, 2002).

Os eosinófilos contêm proteínas que se ligam e lesionam a membrana de parasitas. São responsáveis pelo mecanismo de defesa contra os estágios larvários de parasitas. Além disso, estão envolvidos na modulação de reações alérgicas inflamatórias e de imunocomplexo (THRALL; WEISER, 2007). Possuem habilidade em fagocitar partículas, mas, quando comparados aos neutrófilos, essa habilidade é limitada (SODIKOFF, 2001). Em áreas de inflamação alérgica, os eosinófilos são atraídos pela histamina liberada dos basófilos e mastócitos, migrando para o local inflamado, onde seus grânulos acidófilos liberam conteúdo citotóxico para o meio extracelular e compõem sua atividade defensiva juntamente com a ação fagocitária de complexos antígeno-anticorpo (SINK; FELDMAN, 2006). 
Os linfócitos são as células que especificamente reconhecem e respondem aos antígenos estranhos e formam o centro do sistema imunológico. Estão presentes como células circulantes no sangue ou na linfa, como coleções de células nos órgãos linfoides ou como células dispersas em outros tecidos (CALDER et al., 2002). Os linfócitos maduros se encaixam em duas populações principais: células T e células $\mathrm{B}$, dependendo em qual órgão linfoide primário ocorre o amadurecimento. As células T amadurecem no timo, já as células B podem amadurecer dentro de vários órgãos diferentes, como Bursa de Fabricius nas aves, medula óssea nos primatas e roedores, e placas de Peyer nos ruminantes e suínos (TIZARD, 2002). A diferenciação dos linfócitos B e T, feita por meios imunocitoquímicos, é impossível de ser vista no esfregaço sanguíneo, já que ambas as células são morfologicamente indistinguíveis (GARCIA-NAVARRO, 2005).

Em resposta ao estímulo antigênico, os linfócitos $T$ secretam citocinas, pequenas moléculas proteicas cuja função é promover a proliferação dos linfócitos, ativação de outros tipos celulares, incluindo os linfócitos B, células NK (natural killer) e macrófagos. Os linfócitos B se diferenciam em plasmócitos e produzem anticorpos que fazem parte da resposta imunológica humoral (CALDER et al., 2002).

Os lipídios da dieta podem modificar a concentração plasmática de lipídios, e com isso, as funções de linfócitos e também de macrófagos. A ingestão de altas quantidades de lipídios resulta na diminuição das funções imunológicas, em comparação com dietas pobres em lipídios, por induzir alterações na composição dos fosfolipídios da membrana plasmática e consequentes modificações nas propriedades físicas destas células, como fluidez de membrana, alterando a capacidade das células em responder aos estímulos (CALDER et al., 2002). Assim, a suplementação com lecitina de soja pode afetar a concentração plasmática de lipídios e modular a composição e as funções das células de defesa (MIRANDA, 2005).

Dessa forma, este trabalho teve o objetivo de investigar se a adição de lecitina de soja na ração de cães afeta os parâmetros hematológicos (eritrograma, leucograma), servindo como um estudo inicial para introdução deste suplemento na dieta para a espécie.

\section{MATERIAL E MÉTODOS}

Foram avaliados 8 cães da raça Beagle, pertencentes ao Laboratório de Estudos em Nutrição Canina (LENUCAN) da UFPR, adultos, machos e fêmeas, com pesos médios de 13,410 \pm 0,210 kg, vacinados, desverminados e clinicamente sadios, divididos aleatoriamente em 2 grupos: grupo controle (C) e grupo suplementado com $2 \mathrm{~g} / \mathrm{kg}$ de peso corporal de lecitina de soja (LS) na ração. Os animais eram alimentados 2 vezes ao dia e a água era fornecida ad libidum. O período experimental foi de 60 dias.

As coletas de sangue foram feitas aos dias 1, 30 e 60 . Foram coletados $3 \mathrm{ml}$ de sangue por meio de venopunção jugular. As amostras foram acondicionadas em frascos contendo EDTA (ácido etilenodiamino tetra-acético) a 10\%. As contagens totais de eritrócitos e de leucócitos foram realizadas com auxílio do contador automático de células CC-530 (Cellm®). A contagem diferencial de leucócitos foi realizada em esfregaços sanguíneos utilizando o kit corante Panótico Rápido (Laborclin).

A estatística descritiva foi realizada utilizando o programa estatístico Estat ${ }^{\circledR}$, versão 2.0. As comparações entre os grupos foram realizadas empregando-se o esquema fatorial $2 \times 2 \times 3$ (tratamento, sexo, tempo). Os dados foram submetidos à ANOVA e as médias comparadas pelo Teste de Tukey. O delineamento foi inteiramente casualizado. As diferenças foram consideradas significativas para valores de $\mathrm{p}<0,05$.

\section{RESULTADOS E DISCUSSÃO}

A contagem das células sanguíneas nos grupos está mostrada na Tabela 1. Houve diminuição significativa do número de monócitos, nos primeiros 30 dias, e do número de bastonetes, no último mês do experimento no grupo suplementado. Entretanto, como o grupo controle também apresentou essas mesmas alterações, foi concluído que se devem a mudanças no ambiente, as quais prejudicaram todos os cães durante o período experimental, como estresse por dominância, tanto entre os machos, como entre as fêmeas, infestação por pulgas e otite externa, sem relação com o suplemento. De acordo com 
Jain (1993), a diminuição do número de bastonetes não tem significado clínico, em face de sua ampla faixa de ocorrência ( 0 a 300 / uI), já a monocitopenia transitória pode ser vista em estágios iniciais de estresse; mas mesmo com essa variação, as contagens celulares dos animais estão dentro dos padrões considerados normais pelo autor. Não foram encontradas diferenças nas demais contagens de células sanguíneas entre o grupo teste e o grupo controle.

Segundo Dhabhar e McEwen (1997), em situações de estresse agudo, ocorre uma estimulação do sistema imunológico, já em condições de estresse crônico, o sistema imunológico sofre supressão. Em diferentes espécies de mamíferos, a contagem absoluta e relativa de neutrófilos segmentados tende a aumentar durante o estresse agudo, como observado em humanos (ISOWA et al., 2003; ZORRILA et al., 2001), em macaco rhesus (MORROW-TESCH; McGLONE; NORMAN, 1993), em macaco-decheiro (COE; HALL, 1996) e em hamsters (BILBO et al., 2002).

Além do estresse, é sabido que a quantidade de lipídios e os tipos de ácidos graxos presentes na dieta também podem afetar as funções do sistema imunológico. A deficiência de ácidos graxos essenciais na dieta pode reduzir a imunidade mediada por células. Os linfócitos são as principais células envolvidas nas respostas imunológicas adaptativas e a composição de ácidos graxos destas células e de todas as outras do sistema imunológico é alterada de acordo com os lipídios da dieta, consequentemente alterando a capacidade destas células em produzir substâncias envolvidas na imunorregulação, como os eicosanoides (CALDER et al., 2002).

A influência dos lipídios da dieta sobre as células do sistema imunológico também pode resultar da alteração na síntese de mediadores químicos formados a partir dos fosfolipídios de membrana, que poderiam reduzir a afinidade de substratos para enzimas que sintetizam moléculas de sinalização, uma vez que alterações na composição dos fosfolipídios da membrana plasmática e consequentes modificações nas propriedades físicas dessas células, como fluidez de membrana ou de regiões específicas da membrana, alteram a habilidade da célula em responder estímulos (CALDER, 2001).

Após 24 meses de suplementação com lecitina oral, Tompkins e Parkin (1980) não observaram modificações no perfil hematológico de indivíduos sadios. Em grupos de roedores sadios e diabeticamente induzidos, Miranda (2005) verificou que a suplementação com lecitina de soja na dieta durante 7 dias não afetou a contagem das células brancas do sangue.

Observamos também diferenças nas contagens de leucócitos totais e neutrófilos segmentados entre os sexos (Tabela 1). As fêmeas apresentaram maior contagem dessas células. Em primatas, Cunha et al. (2005) não observaram diferenças entre os sexos, na contagem de neutrófilos e leucócitos totais.

TABELA 1 - Médias das contagens de células sanguíneas nos grupos experimentais, durante o período de estudo

\begin{tabular}{|c|c|c|c|c|c|c|c|}
\hline Fator & $\begin{array}{l}\text { Hemácias } \\
\text { (milhões/ul) }\end{array}$ & $\begin{array}{l}\text { Leucócitos } \\
\text { (/ul) }\end{array}$ & $\begin{array}{c}\text { Segmentados } \\
(/ \mathrm{ul})\end{array}$ & $\begin{array}{c}\text { Bastonetes } \\
\text { (/ul) }\end{array}$ & $\begin{array}{c}\text { Monócitos } \\
\text { (/ul) }\end{array}$ & $\begin{array}{l}\text { Linfócitos } \\
\text { (/ul) }\end{array}$ & $\begin{array}{c}\text { Eosinófilos } \\
\text { (/ul) }\end{array}$ \\
\hline Controle & 7,14 & 9358,33 & 6153,83 & 108,33 & 528,33 & 2348,17 & 244,67 \\
\hline Lecitina & 6,94 & 8783,33 & 5692,17 & 180,08 & 629,83 & 1991,67 & 289,58 \\
\hline Macho & 7,14 & $8550^{\mathrm{B} *}$ & $5317,58^{\mathrm{B}^{*}}$ & 154,25 & 546,92 & 2331,17 & 225,08 \\
\hline Fêmea & 6,94 & $9591,67^{A^{*}}$ & $6528,42^{\mathrm{A}^{*}}$ & 134,17 & 611,25 & 2008,67 & 309,17 \\
\hline Dia 01 & 7,43 & 9537,5 & 6522,5 & $145,25^{\mathrm{A}^{*}}$ & $764,75^{A^{*}}$ & 1833,88 & 271,13 \\
\hline Dia 30 & 6,81 & 8325 & 5194,75 & $239,75^{A^{*}}$ & $360,63^{\mathrm{B}^{*}}$ & 2294 & 235,88 \\
\hline Dia 60 & 6,88 & 9350 & 6051,75 & $47,63^{\mathrm{B}^{*}}$ & $611,88^{\mathrm{AB}^{*}}$ & 2381,88 & 294,38 \\
\hline $\mathrm{s}^{1}(\%)$ & 0,68 & 10,60 & 6,13 & 28,14 & 0,56 & 51,51 & 18,93 \\
\hline $\mathrm{CV}^{2}(\%)$ & 4,15 & 14,15 & 11,99 & 46,58 & 7,75 & 23,74 & 22,65 \\
\hline P & $\mathrm{ns}^{3}$ & $<0,05$ & $<0,05$ & $<0,01$ & $<0,05$ & $\mathrm{~ns}^{9}$ & $\mathrm{~ns}^{9}$ \\
\hline Padrão ${ }^{4}$ & 5,5 a 8,5 & 6000 a 17000 & 3000 a 11500 & 0 a 300 & 150 a 1350 & 1000 a 4800 & 100 a 1250 \\
\hline
\end{tabular}

Letras diferentes indicam diferença dentro da mesma coluna, pelo teste de Tukey.

Devio padrão.

Coeficiente de variação.

Não significativo.

Valores padrão de referência para cães adultos, conforme JAIN (1993). 
Em experimentos para definir valores de referência de células sanguíneas em cães, Hadidian e Pawlowski (1964) demonstraram contagens de eosinófilos significativamente maiores em fêmeas do que em machos da raça Beagle; ao contrário de Brunk (1969), que relatou um maior número de eosinófilos em beagles machos ao comparar com as fêmeas, mas não encontrou diferenças entre sexos na contagem das demais células sanguíneas. Com o mesmo objetivo, Reber et al. (1961) também não observaram diferenças significativas entre os sexos na avaliação do hemograma em cães da raça Beagle.

A suplementação da dieta com lecitina de soja tem sido popularmente usada no tratamento de ateromas, hipercolesterolemia, embolismo gorduroso e para melhorar o perfil lipídico; Fujikawa (1991) reportou que a lecitina em doses diárias de 1,5-2,2g reduziu o colesterol sérico em pacientes ateroscleróticos. Ishinaga, Sugiyama e Mochizuki (1994) mostraram forte correlação inversa entre a ingestão diária de fosfolipídios e o colesterol em mulheres japonesas. Ishida et al. (1988) relataram que a lecitina de soja diminuiu o colesterol total em ratos suplementados, mostrando um papel importante na regulação do metabolismo lipídico. Evans et al. (2007) afirmaram que a lecitina de soja na dose de $20 \mathrm{~g} /$ dia melhorou o perfil lipídico de mulheres na fase pós-menopausa. Também nas condições hepáticas induzidas por medicação, álcool, vírus e toxinas, por seu efeito hepatoprotetor (LECHOWSKI et al., 1999; PODOBED et al., 1995); no tratamento da obesidade, pois a lecitina apresenta efeitos sobre o esvaziamento gástrico e ingestão de alimentos. Conforme Nishimukai et al. (2003), ratos recebendo dieta com vários níveis de lecitina apresentaram menores proporções de esvaziamento gástrico e ingestão alimentar, em comparação aos ratos que não receberam suplementação. É recomendada nos casos de alterações de memória e distúrbios mentais (BUCHMAN et al., 2001); para estabilização das membranas celulares, por ser o elemento funcional e estrutural (AABDALLAH; EID, 2004; IPATOVA et al., 2004) e como agente imunomodulador (CALDER, 2001).

Em virtude da escassez de relatos específicos sobre alterações ocorridas no hemograma com o uso da lecitina de soja em animais ou seres humanos em toda a literatura pesquisada, e independente dos resultados encontrados neste estudo, pesquisas sobre seus efeitos em cães são requeridas, já que a lecitina de soja apresenta variadas funções e efeitos múltiplos, tornando seu uso indicado tanto na alimentação e nutrição animal, mantendo a integridade do organismo, assim como na terapia de doenças do metabolismo e do sistema imunitário.

\section{CONCLUSÕES}

Os resultados deste trabalho mostraram que a adição de lecitina de soja na ração de cães sadios durante 60 dias não afetou o perfil hematológico dos animais.

\section{REFERÊNCIAS}

AABDALLAH, D. M.; EID, N. I. Possible neuroprotective effects of lecithin and alpha-tocopherol alone or in combination against ischemia/reperfusion insult in rat brain. Journal of Biochemical and Molecular Toxicology, New York, v. 18, p. 273-278, 2004.

BEVILACQUA, F. et al. Fisiopatologia dos distúrbios hematológicos. In: BEVILACQUA, F. et al. Fisiopatologia clínica. 4. ed. Rio de Janeiro: Atheneu, 1992. p. 523-560.

BILBO, S. D. et al. Short day lengths augment stress-induced leukocyte the trafficking and stressinduced enhancement of skin immune function. Proceeding of the National Academy of Science of the United States of America, Washington, D.C., v. 99, p. 4067-4072, 2002.

BRUNK, R. R. Standard values in the beagle dog: haematology and clinical chemistry. Food and Cosmetics Toxicology, Oxford, v. 7, p. 141-148, 1969. 
BUCHMAN, A. L. et al. Verbal and visual memory improve after choline supplementation in long-term total parenteral nutrition: a pilot study. Journal of Parenteral and Enteral Nutrition, Silver Spring, v. 25, n. 1, p. 30-35, 2001.

CALDER, P. C. et al. Fatty acids and lymphocyte functions. British Journal of Nutrition, London, v. 87, p. 31-48, 2002. Suplemento 1.

CALDER, P. C. The ratio of n-6 to n-3 fatty acids in the diet: impact on T lymphocytes function. European Journal of Lipid Science and Technology, Weinheim, v. 103, p. 390-398, 2001.

CARR, J. H.; RODAK, B. F. Atlas de hematologia clínica. São Paulo: Santos, 2000.

COE, C. L.; HALL, N. R. Psychological disturbance alters thymic and adrenal hormone secretion in parallel but independent manner. Psychoneuroendocrinology, Oxford, v. 21, p. 237-247, 1996.

CUNHA, M. S.; LOPES, D. R.; SOUSA, M. B. C. Variação na contagem de leucócitos em Callithrix jacchus (Linnaeus, 1758) submetidos a uma situação de estresse agudo. Revista Brasileira de Zoociências, Juiz de Fora, v. 7, n. 2, p. 217-229, 2005.

DECLAIR, V. Uso de triglicérides de cadeia média na prevenção de úlceras de decúbito. Revista Brasileira de Enfermagem, Rio de Janeiro, v. 47, n. 2, p. 127-130, 1994.

DHABHAR, F. S.; McEWEN, B. S. Acute stress enhances while chronic stress suppress cell-mediated immunity in vivo: a potential role for leucocytes trafficking. Brain, Behavior and Immunity, Orlando, v. 11, p. 286-306, 1997.

DUNCAN, J. R.; PRASSE, K. W. Patologia clínica veterinária. Rio de Janeiro: Guanabara Koogan, 1982.

EVANS, M. et al. Effect of soy isoflavone protein and soy lecithin on endothelial function in healthy postmenopausal women. Menopause, Hagerstown, v. 14, n. 1, p. 141-149, 2007.

FUJIKAWA, T. Utilization of lecithin and practical functions for dietary use. Yukagaku, Tokyo, v. 40, p. 951-958, 1991.

GARCIA-NAVARRO, C. E. K. Manual de hematologia veterinária. 2. ed. São Paulo: Varela, 2005. GORMLEY, J. J. Brewer's yeast and lecithin: two underrated health promoters. Better Nutrition, El Segundo, v. 59, n. 2, p. 32, 1997.

HADIDIAN, Z.; PAWLOWSKI, A. A. Hematologic, biochemical and organ weight characteristics of beagles. Cancer Chemotherapy Reports, Bethesda, n. 38, p. 17, 1964.

ISHIDA, T. et al. Cholesterol levels and eicosanoid production in rats fed phosphatidylinositol or soybean lecithin. Journal of Nutrition Science and Vitaminology, Tokyo, v. 34, n. 2, p. 237-244, 1988.

ISHINAGA, M.; SUGIYAMA, S.; MOCHIZUKI, T. Daily intakes of fatty acids, sterols and phospholipids by Japanese women and serum cholesterol. Journal of Nutritional Science and Vitaminology, Tokyo, v. 40, p. 557-567, 1994.

ISOWA, T.; H. OHIRA, H.; MURASHIMA, S. Reactivity of immune, endocrine and cardiovascular parameters to active and passive acute stress. Biological Psychology, 2003. No prelo.

IPATOVA, O. M. et al. Biological effects of the soybean phospholipids. Biomeditsinskaia Khimiia, Moskva, v. 50, p. 436-450, 2004.

ITO, L. S. et al. Dietary factors and the risk of gastric cancer among Japanese women: a comparison between the differentiated and non-differentiated subtypes. Annals of Epidemiology, New York, v. 13, p. 24-31, 2003. 
JAIN, N. C. Essentials of veterinary hematology. Philadelphia: Lea \& Febiger, 1993.

LEBLANC, M. J. et al. Effects of dietary soybean lecithin on plasma lipid transport and hepatic cholesterol metabolism in rats. The Journal of Nutritional Biochemistry, Stoneham, v. 14, p. 40-48, 2003.

LECHOWSKI, R. et al. The effect of lecithin supplementation on the biochemical profile and morphological changes in the liver of rats fed different animal fats. Veterinary Research Communications, Dordrecht, v. 23, n. 1, p. 1-14, 1999.

MASTELLONE, I. et al. Dietary soybean phosphatidylcholines lower lipidemia: mechanisms at the levels of intestine, endothelial cell, and hepato-biliary axis. The Journal of Nutritional Biochemistry, Stoneham, v. 11, p. 461-466, 2000.

MILLER, D. L. Health benefits of lecithin and choline. Cereal Foods World, Minneapolis, v. 47, n. 5, p. 178-184, 2002.

MIRANDA, D. T. S. Z. Suplementação da dieta de ratos diabéticos com lecitina de soja: efeitos sobre funções de células do sistema imunitário e sobre concentrações plasmáticas de lipídios. 69 f. Dissertação (Mestrado em Biologia Celular e Molecular) - Universidade Federal do Paraná, Curitiba, 2005.

MORROW-TESCH, J. L.; McGLONE, J. J.; NORMAN, R. L. Consequences of restraint stress on natural killer activity, behavior, and hormone levels in rhesus macaques (Macaca mulatta). Psychoneuroendocrinology, Oxford, v. 18, p. 383- 395, 1993.

NAGATA, C. Ecological study of the association between soy product intake and mortality from cancer and heart disease in Japan. International Journal of Epidemiology, Oxford, v. 29, p. 832 836, 2000.

NAGATA, C. et al. A prospective cohort study of soy product intake and stomach cancer death. British Journal of Cancer, Edinburgh, v. 87, p. 31-36, 2002.

NISHIMUKAI, M.; HARA, H.; AOYAMA, Y. The addition of soybean phosphatidylcholine to triglyceride increases suppressive effects on food intake and gastric emptying in rats. The Journal of Nutrition, Bethesda, v. 133, n. 5, p. 1255-1258, 2003.

PODOBED, O. V. et al. The effect of phosphatidylcholine on repair processes in liver cells in acute CCI4 damage. Voprosy Meditsinskoi Khimii, Moskva, v. 14, p. 13-16, 1995.

REBER, E. F. et al. Standard blood values in the beagle dog. Transactions of the Illinois State Academy of Science, Springfield, v. 54, p. 79, 1961.

SAKAKIMA, Y. et al. Prevention of hepatocarcinogenesis with phosphatidylcholine and menaquinone-4: in vitro and in vivo experiments. Journal of Hepatology, Copenhagen, v. 47, n. 1, p. 83-92, 2007.

SINK, C. A.; FELDMAN, B. F. Urinálise e hematologia laboratorial para o clínico de pequenos animais. São Paulo: Roca, 2006.

SODIKOFF, C. H. White blood cells. In: SODIKOFF, C. H. Laboratory profiles of small animal diseases - a guide to laboratory diagnosis. 3rd ed. Philadelphia: Mosby, 2001. p. 109-124.

STRAYER, L. Bioquímica. 4. ed. Rio de Janeiro: Guanabara Koogan, 1996.

TIZARD, I. R. Órgãos do sistema imunológico. In: TIZARD, I. R. Imunologia veterinária: uma Introdução. 6. ed. São Paulo: Roca, 2002. p. 76-92. 
TOMPKINS, R. K.; PARKIN, L. G. Effects of long-term ingestion of soya phospholipids on serum lipids in humans. The American Journal of Surgery, Belle Mead, v. 140, p. 360-364, 1980. THRALL, M. A.; WEISER, G. Hematologia. In: HENDRIX, C. M. Procedimentos laboratoriais para técnicos veterinários. 4. ed. São Paulo: Roca, 2006. p. 31-78.

Hematologia das espécies domésticas comuns. In: THRALL, M. A. Hematologia e bioquímica clínica veterinária. São Paulo: Roca, 2007. p. 63-198.

WILSON, T. E.; MESERVEY, C. M.; NICOLOSI, R. J. Soy lecithin reduces plasma lipoprotein cholesterol and early atherogenesis in hypercholesterolemic monkeys and hamsters: beyond linoleate. Atherosclerosis, Limerick, v. 140, p. 147-153, 1998.

YONGZHI, J.; SANG, K. N.; SUNG, I. K. Egg phosphatidylcholine decreases the lymphatic absorption of cholesterol in rats. The Journal of Nutrition, Bethesda, v. 131, p. 2358-2363, 2001.

ZHANG, X. et al. Soy food consumption is associated with lower risk of coronary heart disease in Chinese women. The Journal of Nutrition, Bethesda, v. 133, p. 2874-2878, 2003.

ZORRILA, E. P. et al. The relationship of depression and stressors to immunological assays: a metaanalytic review. Brain, Behavior and Immunity, Orlando, v. 15, p. 199-226, 2001.

Recebido: 03/11/2008

Received: 11/03/2008

Aprovado: 20/01/2009

Approved: 01/20/2009 